OPEN ACCESS

Edited by:

Chang H. Kim,

University of Michigan, United States

Reviewed by:

Xin Li,

Henan University of Science and

Technology, China

Alireza Mani,

University College London,

United Kingdom

*Correspondence:

Yanfei Chen

yanfeichen1983@163.com

Lanjuan Li

lili@zju.edu.cn

Specialty section:

This article was submitted to Microbiome in Health and Disease,

a section of the journal

Frontiers in Cellular and Infection

Microbiology

Received: 29 May 2018 Accepted: 16 November 2018 Published: 30 November 2018

Citation:

Chen Y, Guo J, Shi D, Fang D, Chen C and Li L (2018) Ascitic Bacterial

Composition Is Associated With

Clinical Outcomes in Cirrhotic Patients

With Culture-Negative and

Non-neutrocytic Ascites.

Front. Cell. Infect. Microbiol. 8:420.

doi: 10.3389/fcimb.2018.00420

\section{Ascitic Bacterial Composition Is Associated With Clinical Outcomes in Cirrhotic Patients With Culture-Negative and Non-neutrocytic Ascites}

\author{
Yanfei Chen*, Jing Guo, Ding Shi, Daiqiong Fang, Chunlei Chen and Lanjuan Li* \\ State Key Laboratory for Diagnosis and Treatment of Infectious Disease, Collaborative Innovation Center for Diagnosis and \\ Treatment of Infectious Diseases, The First Affiliated Hospital, Zhejiang University, Hangzhou, China
}

Ascites bacterial burden is associated with poor clinical outcomes in patients with end-stage liver disease. However, the impact of ascitic microbial composition on clinical course was still not clear. In this study, the ascitic microbiota composition of 100 cirrhotic patients with culture-negative and non-neutrocytic ascites were researched with 16S rRNA pyrosequencing and enterotype-like cluster analysis.

Results: By characterizing the ascitic microbial composition, two distinct microbial clusters were observed, Cluster 1 (86 patients) and Cluster 2 (14 patients). Cluster 1 showed lower microbial richness than Cluster 2. At the phylum level, Cluster 1 had greater abundance of Bacteroidetes and Firmicutes, but less abundance of Proteobacteria and Actinobacteria than Cluster 2. At the family level, family Bacteroidales S24-7 group, Prevotellaceae, Lachnospiraceae, Lactobacillaceae, Rikenellaceae, and Vibrionaceae were found over-represented in Cluster 1. And family Acetobacteraceae, Erysipelotrichaceae, Rickettsiaceae, and Streptococcaceae were found enriched in Cluster 2. The levels of plasma cytokine IL-17A, IL-7, and PDGF-BB were found significantly higher in Cluster 1 than in Cluster 2 . There were four OTUs closely correlated with plasma cytokines, which were OTU 140 and OTU 271 (both from Bacteroidales S24-7 group), OTU 68 (Veillonellaceae), and OTU 53 (Helicobacteraceae). Patients from Cluster 1 showed significant higher short-term mortality than patients from Cluster 2.

Conclusion: Our study demonstrated that the microbial composition of culture-negative and non-neutrocytic ascites in cirrhotic patients is associated with short-term clinical outcomes. The results here offer a rational for the identification of patients with high risk, and provide references for selective use of prophylactic methods.

Keywords: ascitic fluids, microbiome, end-stage liver disease, bacterial translocation, cytokines 


\section{INTRODUCTION}

Bacterial translocation (BT) is increasingly recognized as a key driver in the development of complications in end-stage liver disease (Wiest et al., 2014). BT can cause infections, in particular, spontaneous bacterial peritonitis (SBP). In the absence of overt infection, BT may further stimulate the immune system and contribute to hemodynamic alterations and complications. The accepted pathogenic theory of BT postulates that bacteria escape from the intestinal lumen and reach the mesenteric lymph nodes by crossing the intestinal, subsequently disseminating to the bloodstream and the ascitic fluid (AF). The presence of bacteria in AF has been investigated as a simple way for studying BT in cirrhosis.

Selective decontamination of the digestive tract (SDD) can prevent $\mathrm{BT}$ and reduce severe infections and mortality in patients with end stage liver disease (Llovet et al., 1996). SDD prevents secondary bacterial colonization through application of nonabsorbable antimicrobial agents in the gastrointestinal tract (De Smet et al., 2009). SDD is a widely evaluated but highly controversial intervention. One of the major concern about SDD is the development of bacterial resistance (Wunderink, 2010). Besides, SDD targets at both normal bacteria and potential pathogenic bacteria. The use of SDD might disturb the gut microbial balance and cause bad consequences. Hence, the identification of patients with high risk of short-term mortality and infections could provide a rational for selective use of SDD.

By culture, only a minority of ascitic bacteria can be isolated, even in the presence of overt infection. Using cultureindependent techniques, Such et al. reported that bactDNA can be commonly detected in culture-negative and non-nuetrocytic $\mathrm{AF}$ (Such et al., 2002). It was further confirmed by the same group that the presence of bactDNA in patients with cirrhosis during an ascitic episode is an indicator of poor prognosis, which may be related to development of acute-on-chronic liver failure at short term (Zapater et al., 2008). Fagan et al. found that ascites bacterial burden and immune cell profile are associated with poor clinical outcomes in the absence of overt infection (Fagan et al., 2015).

With more sensitive molecular techniques such as $16 \mathrm{~S}$ rRNA and shotgun metagenomic pyrosequencing, depth profile of microbial communities in AF have been characterized in small sample sizes recently (Rogers et al., 2013; Feng et al., 2015). Using $16 \mathrm{~S}$ rRNA gene pyrosequencing, Rogers et al. found that differences in structure and membership of AF microbial communities correlated with severity of liver cirrhosis (Rogers et al., 2013). In their research, propidium monoazide treatment were applied to ascitic samples to characterize only viable bacteria. However, the composition of translocated bacteria, both viable and non-viable, might worth studying. Circulation bacterial fragments are found with pathological significance in patients with inflammatory bowel disease (Gutiérrez et al., 2016), end stage disease (Zapater et al., 2008), and chronic hemodialysis

Abbreviations: BT, bacterial translocation; SBP, spontaneous bacterial peritonitis; AF, ascitic fluids; SDD, selective decontamination of the digestive tract; OTU, operational taxonomic unit; $\mathrm{CH}$, Calinski-Harabasz index; HR, hazard ratio; $95 \%$ CI, $95 \%$ confidence interval of hazard ratio.
(Bossola et al., 2009). Grade of soluble inflammatory response is mainly affected by plasmatic concentration of bactDNA. And, differences in inflammatory responses were observed between gram negative and gram positive bacterial fragment translocation (Caro et al., 2016).

The aim of this prospective study has been to assess the associations between $\mathrm{AF}$ microbial composition and clinical outcomes in cirrhotic patients with culture-negative and nonneutrocytic ascites. We applied a combination of $16 \mathrm{~S}$ rRNA pyrosequencing and enterotype-like cluster analysis to identify AF microbial clusters. The resulting AF microbial clusters were correlated with plasma cytokine profiles and clinical outcomes. This fact may become a relevant clinical issue since it provides a reference for identification of high risk patients, who need prophylactic SDD.

\section{MATERIALS AND METHODS}

\section{Patients}

AF were obtained from 100 consecutive cirrhotic patients undergoing clinically indicated therapeutic or diagnostic paracentesis for ascites at the First Affiliated Hospital of Zhejiang University. Cirrhosis was diagnosed by histology or by clinical, laboratory, and/or image findings. Inclusion criteria were the presence of cirrhosis and ascites fluids. Exclusion criteria were infected AF with positive culture or $>250$ polymorphonuclear, upper gastrointestinal bleeding, intake of antibiotics in previous 2 weeks including norfloxacin as prophylaxis of SBP, two or more of criteria of systemic inflammatory response syndrome (temperature $>38^{\circ} \mathrm{C}$ or $<3^{\circ} \mathrm{C}$, heart rate $>90$ beats $/$ min, respiratory rate $>20$ breaths $/ \mathrm{min}$, blood white blood cells $<4,000$ or $>12,000 / \mathrm{mm}^{3}$ ) (Rangel-Frausto et al., 1995).

AF samples were obtained when a large volume paracentesis were needed as a part of the patient's treatment. Paracentesis were performed under aseptic conditions following the usual procedures. AF samples for routine biochemical study were obtained. Blood samples of the same day was obtained for hematological, biochemical, coagulation, and cytokine profile analysis. Both blood and AF samples were kept under aseptic conditions. All the patients in study were followed up for 90 successive days. The occurrence of death and complications during the 90 days were recorded. One patient from Cluster 1 underwent liver transplant. The patient was so critically ill that would not survive without liver transplant. We classified the patient as non-survivors. And the day of liver transplant was used to calculate the survival time.

\section{DNA Extraction and 16S rRNA Sequencing}

$\mathrm{AF}$ were stored at $-80^{\circ} \mathrm{C}$ immediately after collected. Total bacterial DNA was extracted from AF samples using QIAamp DNA Mini Kit (Qiagen, Valencia, CA) according to manufacture's instruction. Bacterial $16 \mathrm{~S}$ rRNA V3-V4 region was amplified using the 343F/798R primer set (343F 5'-TACGGRAGGCAGCAG-3', 798R 5'AGGGTATCTAATCCT- $3^{\prime}$ ). PCR reaction was performed using phusion high-fidelity PCR Mastermix (Invitrogen, Carlsbad, CA, USA) with the following condition: $95^{\circ} \mathrm{C}$ for $3 \mathrm{~min}$ ( 1 cycle), 
$95^{\circ} \mathrm{C}$ for $30 \mathrm{~s} / 55^{\circ} \mathrm{C}$ for $30 \mathrm{~s} / 72^{\circ} \mathrm{C}$ for 30 s ( 35 cycles), $72^{\circ} \mathrm{C}$ for $10 \mathrm{~min}$. PCR product was purified using Agencourt AMPure XP beads (Beckman coulter, Brea, CA) according to manufacture's protocol. Pyrosequencing was conducted on an Illumina Miseq 2*300 platform according to protocols.

\section{Pyrosequencing Data Bioinformatics Analysis}

Raw reads were filtered according to length and quality criteria. Filter-pass reads were assembled. After assembly, chimeric sequences were removed using the Usearch software based on the Uchime algorithm (Edgar et al., 2011). Operational Taxonomic Unit (OTU) was picked using de novo OTU picking protocol with a $97 \%$ similarity threshold. Taxonomy assignment of OTUs was performed by comparing sequences to Greengenes. Enterotypelike clustering was performed in R with package "BiotypeR" on Jensen-Shannon distance for the OTU-level relative abundance profile (Arumugam et al., 2011). The optimal number of clusters was chosen based on Calinski-Harabasz $(\mathrm{CH})$ values. To determine compositional features that were differentially abundant either between clusters, LEfSe was applied (Segata et al., 2011). The R package "phyloseq" was used for alpha diversity analysis (McMurdie and Holmes, 2013).

\section{Cytokine and Chemokine Measurements}

The plasma levels of 27 cytokines and chemokines were measured using the Bio-Plex ProTM Human Cytokine Array 27-Plex Group I on a Luminex $200^{\mathrm{TM}}$ (Luminex ${ }^{\circledR}$ Multiplexing Instrument, Merck Millipore) following the manufacturers' instructions. Then, we analyzed the raw data using xPONENT 3.1 software (Merck Millipore). We assumed a value of $0.1 \mathrm{pg} / \mathrm{mL}$ for statistical purposes in cases in which the concentration was undetectable.

\section{Statistical Analysis}

Two-sided student's $t$-test and Mann-Whitney $U$-tests was used to determine whether the differences in the alpha-diversity, cytokine, and chemokine levels between groups were statistically significant. We used Spearman's rank correlation coefficient analysis to analyze the linear correlation. The Benjamini \& Hochberg method was used to control the false discovery rate for multiple testing corrections. Cox proportional hazards models were used to evaluate the association between variables and the 90 -day outcome. A multivariable stepwise logistic regression test was used to evaluate the independent clinical parameters predicting mortality. The statistical tests and plotting were done in R with package "plyr," "ggplot2."

\section{RESULTS}

\section{Clinical Characteristics and Pyrosequencing Data Summary}

A total of 100 cirrhotic patients were included in this AF microbial profiling study. Of the patients, 56 patients were Hepatitis-B-virus related, 15 patients were alcoholic cirrhosis, 3 patients were primary biliary cirrhosis, and other 26 patients were cryptogenic cirrhosis. Of all the patients, a total of 29 patients died in 90 days, with a short term mortality of $29 \%$. A total of $1,595,225$ high-quality sequences were produced, accounting for $98.1 \%$ of valid sequences (average sequence length $419 \mathrm{bp}$ ).

\section{Two Clusters Identified for Ascitic Microbiome}

The partitioning around medoids method using Jensen-Shannon distance for the OTUs-level relative abundance profile was used to investigate whether AF microbiota can be classified into clusters. The Silhouette index for two clusters was 1.86, which indicates strong support of two clusters. The two clusters were visualized by between class analyze, and showed clear separations (Figure 1A). Two clusters were confirmed with the highest $\mathrm{CH}$ value, as the optimal number of clusters (Figure 1B).

When comparing the diversity index, Cluster 1 were found to have significantly lower richness than Cluster 2. After the sequencing depth normalized, the observed OTUs in Cluster 1 was significantly lower than in Cluster $2(133 \pm 53$ vs. $190 \pm 49$, $p=0.001)$ (Figure 1C), as well as the index of Chao 1 (156 \pm 69 vs. $206 \pm 53, p=0.004$ ) (Figure 1D).

\section{Compositional Analysis of AF Microbial Clusters}

To identify signature taxa, we tested for significant differences among taxa displaying $>1 \%$ relative abundance in the whole dataset (Figure 2). Major differences were observed between Cluster 1 and Cluster 2. At the phylum level (Figure 2A), Bacteroidetes and Firmicutes were found over-represented in Cluster 1, while Proteobacteria and Actinobacteria were found over-represented in Cluster 2.

At the class level (Figure 2B), Bacteroidia, Bacilli, Clostridia, and Gammaproteobacteria were found with significant higher relative abundance in Cluster 1 than in Cluster 2. And, Alphaproteobacteria, Erysipelotrichia, Actinobacteria, and Coriobacteria were enriched in Cluster 2.

At the family level (Figure 2C), Bacteroidia was linked to increased S24-7, Prevotellaceae and Rikenellaceae, while Clostridia expansion was linked to increased Lachnospiraceae in Cluster 1. Besides, Lactobacillaceae and Vibrionaceae were also found enriched in Cluster 1. The expansion of Alphaproteobacteria in Cluster 2 was linked to increased Acetobacteraceae, LD12-freshwater group, Rickettsiaceae, and A0839, while Gammaproteobacteria abundance was linked to increased Moraxellaceae.

At the genus level (Figure 2D), bacterial genus found overrepresented in Cluster 1 included Lactobacillus, Lachnospiraceae NK4A136 group, Alloprevotella, Vibrio, and Prevotella. Bacterial genus Saccharibacter, Commensalibacter, Erysipelotrichia, Allobaculum, Rickettsia, and Cupriavidus were found enriched in Cluster 2.

\section{Plasma Cytokine and Chemokine Levels Correlated With the AF Microbial Compositions}

We performed multiples analyses of 27 cytokine and chemokine mediators using the plasma samples collected from the cirrhotic 

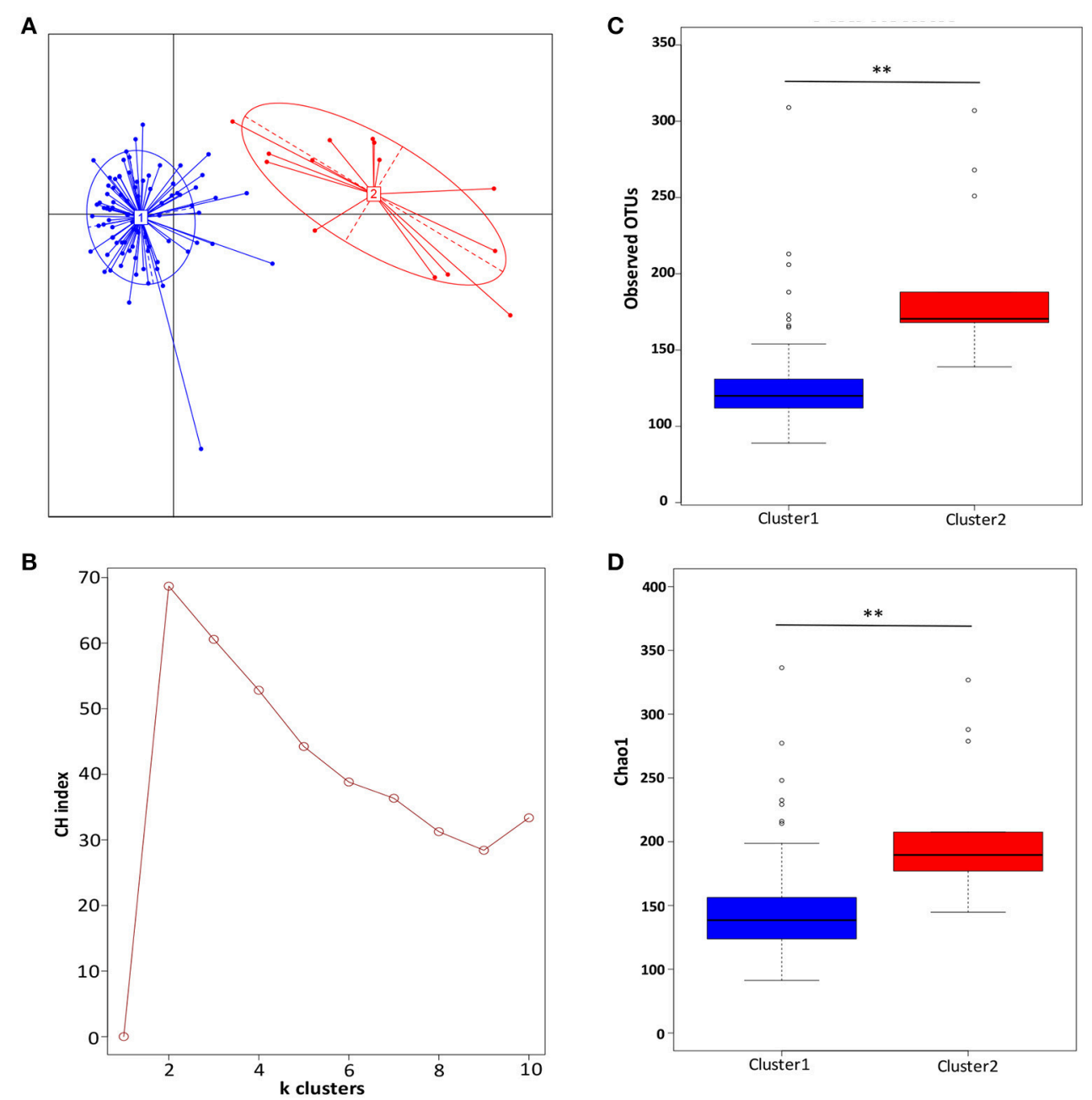

FIGURE 1 | Two clusters were observed in ascitic fluids microbiota. (A) The principal coordinate analysis of the Jensen-Shannon distance generated from the OTU-level relative abundance profiles. Samples are colored by clusters identified by the partitioning around medoids clustering algorithm. Dark blue, Cluster 1; red, Cluster 2. (B) Two clusters were supported with the highest Calinski-Harabasz (CH) pseudo F-statistic value, as the optimal number of clusters. (C) Boxplot comparison of the number of observed OTUs between Cluster 1 and Cluster 2. (D) Boxplot comparison of the Chao1 index between Cluster 1 and Cluster $2 .{ }^{* \star} p<$ 0.01 based upon Mann-Whitney U-tests with Benjamini \& Hochberg correction.

patients. The plasma cytokine and chemokine profile was measured in 78 patients ( 68 patients of Cluster 1 and 10 patients in Cluster 2). The level of plasma cytokine PDGF-BB was found significantly higher in patients of Cluster 1 than in patients of Cluster $2(12.5 \pm 18$ vs. $5.2 \pm 4.2, p=0.006)$. Plasma cytokine IL-7 (6.39 \pm 6.31 vs. $3.96 \pm 1.87, p=0.015)$ and IL-17A (9.03 \pm 29.72 vs. $0.35 \pm 0.77, p=0.019$ ) were also found significantly higher in patients of Cluster 1 than in patients of Cluster 2 (Figure 3).

At the OTU level, there were 14 OTUs correlated with plasma cytokines (Figure 4). The 14 OTUs were from six bacterial families, including S24-7 group (6 OTUs), Lachnospiraceae (2 OTUs), Rikenellaceae (2 OTUs), Veillonellaceae (2 OTUs), Helicobacteraceae (1 OTU), and Prevotellaceae (1 OTU). There were four OTUs closely correlated with multiple cytokines, which were OTU 140 and OTU 271 (S24-7 group), OTU 68 (Veillonellaceae), and OTU 53 (Helicobacteraceae).

\section{Cluster 1 Showed Higher Mortality Rate but Lower Incidence of SBP}

Among the 100 patients in the study, a total of 86 patients $(86 \%)$ were classified as AF Cluster 1, while other 14 patients (14\%) fell into AF Cluster 2. All the patients in study were followed up for 90 successive days for occurrence of death and complications. For patients of AF Cluster 1, 29 patients died in 90 days, with a short term mortality of $33.7 \%$. And in patients of AF Cluster 2 , no patients died in 90 days. The short term mortality was significantly higher in Cluster 1 than in Cluster $2(P=0.01)$ (Table 1). However, the incidence of SBP was found slightly higher in Cluster 2 than in Cluster $1(p=0.06)$. Among the 86 patients in Cluster 1, only 27 patients had SBP in 90 days, with a prevalence of $31.4 \%$. And in Cluster 2, 8 patients were diagnosed SBP in 90 days, with a prevalence of $57.1 \%$. The severity of disease, as estimated by MELD score or Child Pugh score, was comparable between Cluster 1 and Cluster 2 . 
A

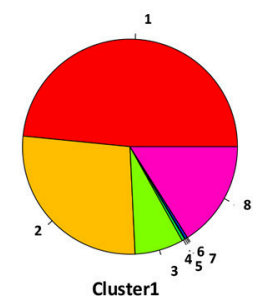

1 Bacteroidetes
$\square \quad 2$ Firmicutes

2 Firmicutes
3 Proteobacteria

3 Proteobacteria
4 Fusobacteria

든 5 Tenericutes

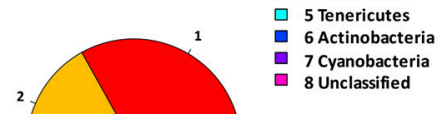

8 Unclassified

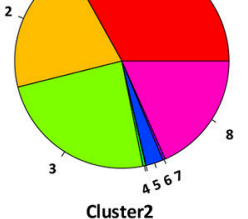

Cluster2

B
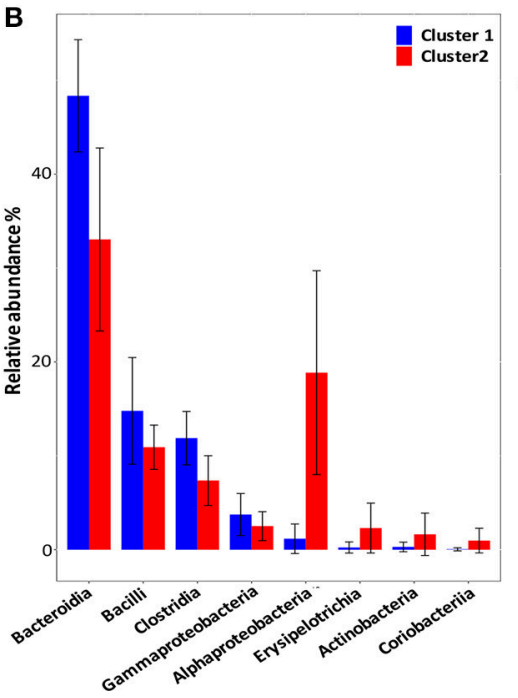

C

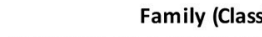

Bacteroidales_S24_7_group (Bacteroidia)

Prevotellaceae (Bacteroidia)

Lachnospiraceae (Clostridia)

Lactobacillaceae (Bacilli)

Rikenellaceae (Bacteroidia)

Vibrionaceae (Gammaproteobacteria)

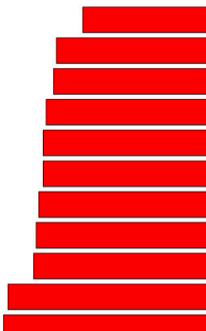

Streptococcaceae (Bacilli)

A0839 (Alphaproteobacteria)

Microbacteriaceae (Actinobacteria)

Sporichthyaceae (Actinobacteria)

Coriobacteriaceae (Coriobacteriia)

Moraxellaceae (Gammaproteobacteria)

Burkholderiaceae (Betaproteobacteria)

Rickettsiaceae (Alphaproteobacteria)

Erysipelotrichaceae (Erysipelotrichia)

LD12_freshwater_group (Alphaproteobacteria)

Acetobacteraceae (Alphaproteobacteria)

D

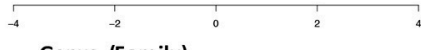

Genus (Family)

Lactobacillus (Lactobacillaceae)

uncultured_Bacteroidales_bacterium (Bacteroidales_S24_7_group)

Lachnospiraceae_NK4A136_group (Lachnospiraceae)

uncultured_bacterium (Bacteroidales_S24_7_group)

Alloprevotella (Prevotellaceae)
Vibrio (Vibrionaceae)

Prevotella_1 (Prevotellaceae)

Alistipes (Rikenellaceae)

Prevotella_7 (Prevotellaceae)

Pseudobutyrivibrio (Lachnospiraceae

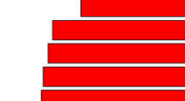

tococcus (Streptococcaceae)

Ambiguous_taxa (A0839)

Candidatus_Aquiluna (Microbacteriaceae)

hgcl_clade (Sporichthyaceae)

hgcl_clade (Sporichthyaceae)

Coriobacteriaceae_UCOO2 (Cor

Acinetobacter (Moraxellaceae)

Cupriavidus (Burkholderiaceae)

Rickettsia (Rickettsiaceae)

Allobaculum (Erysipelotrichaceae)

Commen sali ibacter (Acetobacteraceae)

uncultured_bacterium (LD12_treshwater_group)

Saccharibacter (Acetobacteraceae)

FIGURE 2 | Compositional analysis of ascitic microbial clusters. (A) Pie chart comparison of bacterial phyla represented in two clusters (upper: Cluster 1, lower: Cluster 2). (B) Histograms showing differentially enriched bacterial classes between Cluster 1 and Cluster 2. Blue histograms, Cluster 1; red histograms, Cluster 2. (C). LEfSe analysis revealed differentially enriched bacterial families associated either with Cluster 1 (blue) or Cluster 2 (red). (D) LEfSe analysis revealed differentially enriched bacterial genus associated either with Cluster 1 (blue) or Cluster 2 (red).

Cox regression analysis was used to investigate potential predictors of mortality. The Kaplan-Meier curves for the 90day survival of different AF clusters are shown in Figure 5. Although the 90-day survival rate showed significant difference between Cluster 1 and Cluster $2(P=0.02)$. Univariate logistic regression analysis demonstrated that the AF Cluster 1 was not a significant predictor of 90 -day mortality $(P=0.132)$. Cox univariate analyses indicated several plasma cytokines (TNF- $\alpha$, IL-15, IL-2, IL-1 $\beta$, and IL-12P70) and MELD score as predictors of 90-day mortality. Using multivariate analysis, MELD score (HR 1.075, 95\% CI 1.021-1.132, $P=0.006$ ) and plasma TNF$\alpha$ level (HR 1.001, 95\% CI 1.000-1.001, $P=0.008$ ) were found independent predictors of 90-day mortality (Table 2).

\section{DISCUSSION}

Bacterial translocation is thought as a major mechanism of complications and mortality in end-stage liver disease. Previous studies have confirmed presence of bacterial DNA in ascites fluid, even in culture-negative and non-neutrocytic ascites. Also, positive associations between ascites bacterial burden and poor clinical outcomes was observed (Fagan et al., 2015). This study demonstrated that not only the quantity of bacteria in AF but also the composition could impact the clinical outcomes of end-stage liver disease. The possible mechanism might be associated with system immune responses and cytokine expressions.

Based on the microbial composition of AF, the patients could be classified into two clusters. Cluster 1 has high mortality, and Cluster 2 has high prevalence of SBP. The group with higher mortality had more Bacteroidetes and Firmicutes than another group. Firmicutes and Bacteroidetes are the two most dominant bacterial phyla in human intestines (Turnbaugh et al., 2009). The translocation of these commensal bacteria into the abdominal cavity might indicate severe damage of intestinal mucosa barrier, which usually lead to poor outcomes. Structural and functional alterations in the intestinal mucosa 


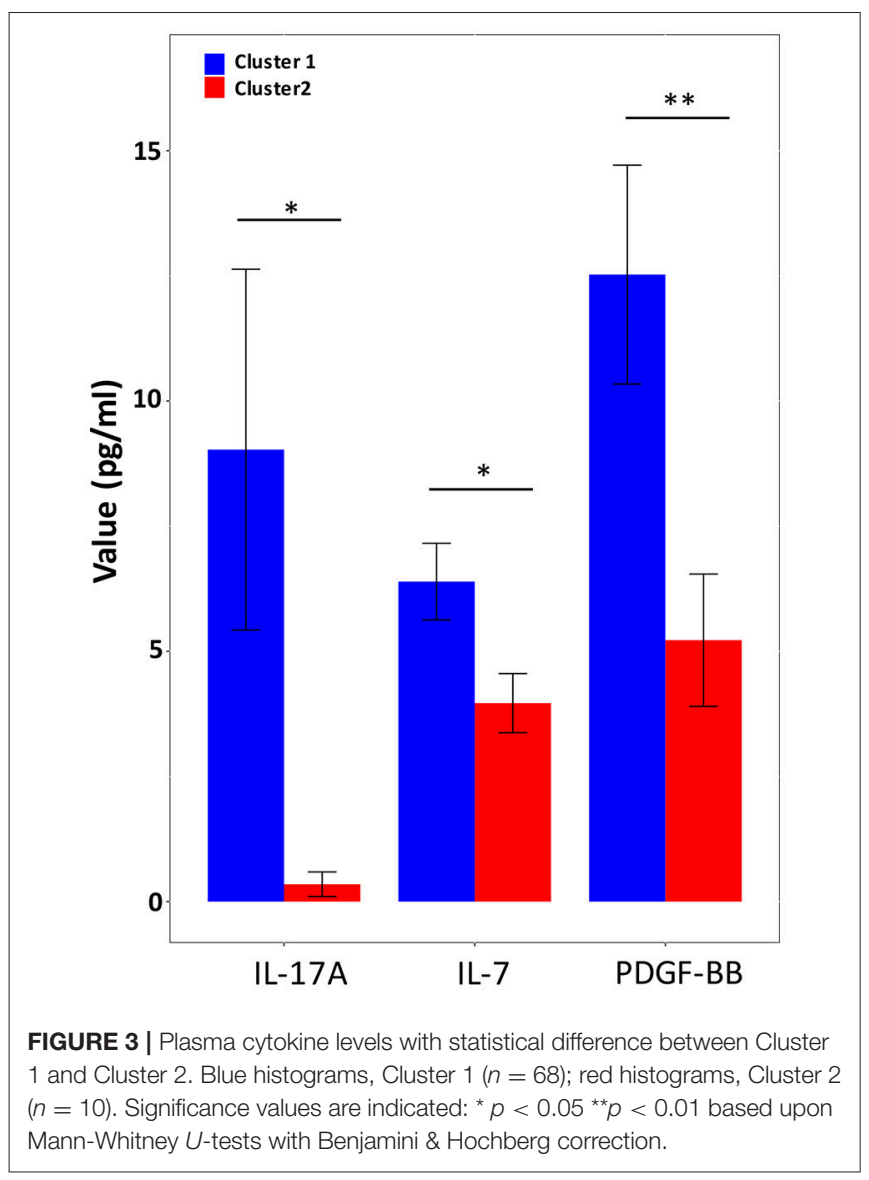

that increase intestinal permeability to bacteria and its products have been described in cirrhosis (Bellot et al., 2013). Altered gut microbiota profile is associated with high intestinal permeability, including less abundant of Ruminococcaceae and more abundant of Lachnospiraceae in subjects with high intestinal permeability compared with subjects with low intestinal permeability (Leclercq et al., 2014). Accordingly, it was observed here that ascitic microbiota of Cluster 1 have more abundance of Lachnospiraceae than that of Cluster 2. The findings add to the evidence that BT from the leaky gut participate in the progress of liver cirrhosis.

Our results showed over-represented of Alphaproteobacteria and Actinobacteria in AF of Cluster 2. Both Alphaproteobacteria and Actinobacteria are common members of marine and freshwater bacterioplankton assemblages. Genomic analysis demonstrated the genome characteristics of the bacterioplankton that render them to well-adapted to such nutrient and energylimited conditions (Salcher et al., 2011). One possible reason is that the intestinal permeability of patients in Cluster 2 is not severely damaged as patients in Cluster 1, and bacteria which are able to translocate to AF need greater ability to survive in nutrient-limited circumstances.

Bacterial class Gammaproteobacteria was found with significantly higher relative abundance in Cluster 2 than in Cluster 1. The Moraxellaceae and Acinetabacter were responsible

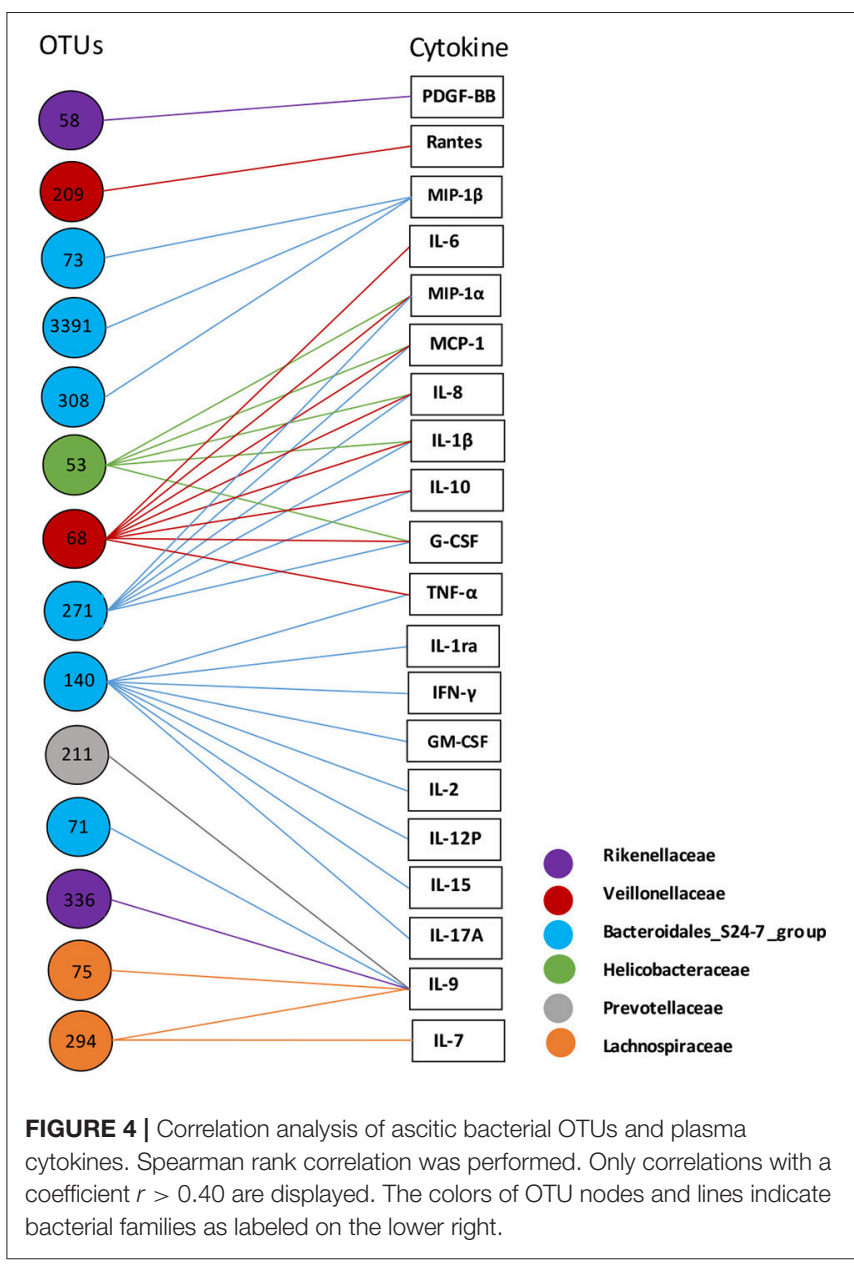

TABLE 1 | Clinical characteristics of patients with different ascitic microbiota clusters.

\begin{tabular}{lccc}
\hline Variable & Cluster $\mathbf{1}(\boldsymbol{n}=\mathbf{8 6})$ & Cluster 2( $\boldsymbol{n}=\mathbf{1 4})$ & $\boldsymbol{p}$-value \\
\hline Age (year) & $56 \pm 11$ & $54 \pm 15$ & 0.7 \\
Gender (M/F) & $16 / 70$ & $4 / 10$ & 0.7 \\
Child-Pugh score & $9 \pm 2$ & $9 \pm 2$ & 0.98 \\
MELD score & $1.45 \pm 0.99$ & $1.43 \pm 0.57$ & 0.81 \\
90-day mortality & $33.7 \%$ & $0 \%$ & 0.01 \\
90-day SBP & $31.4 \%$ & $57.1 \%$ & 0.06
\end{tabular}

SBP, Spontaneous bacterial peritonitis.

for the enrichment of Gammaproteobacteria at the family and genus level, respectively. The incidence of SBP was found slightly higher in Cluster 2 than in Cluster $1(p=0.06)$. Acinetobacter species have become increasingly important nosocomial pathogens worldwide and can result in a wide range of infections, including bacteremia, pneumonia, urinary tract infection, peritonitis, among other (Breslow et al., 2011). In end stage liver disease, Acinetobacter spp. is among the major pathogens that responsible for SBP (Gunjaca and Francetić, 2010). Acinetobacter baumannii and Acinetobacter iwoffii were two major pathogenic species isolated in liver transplant patients 


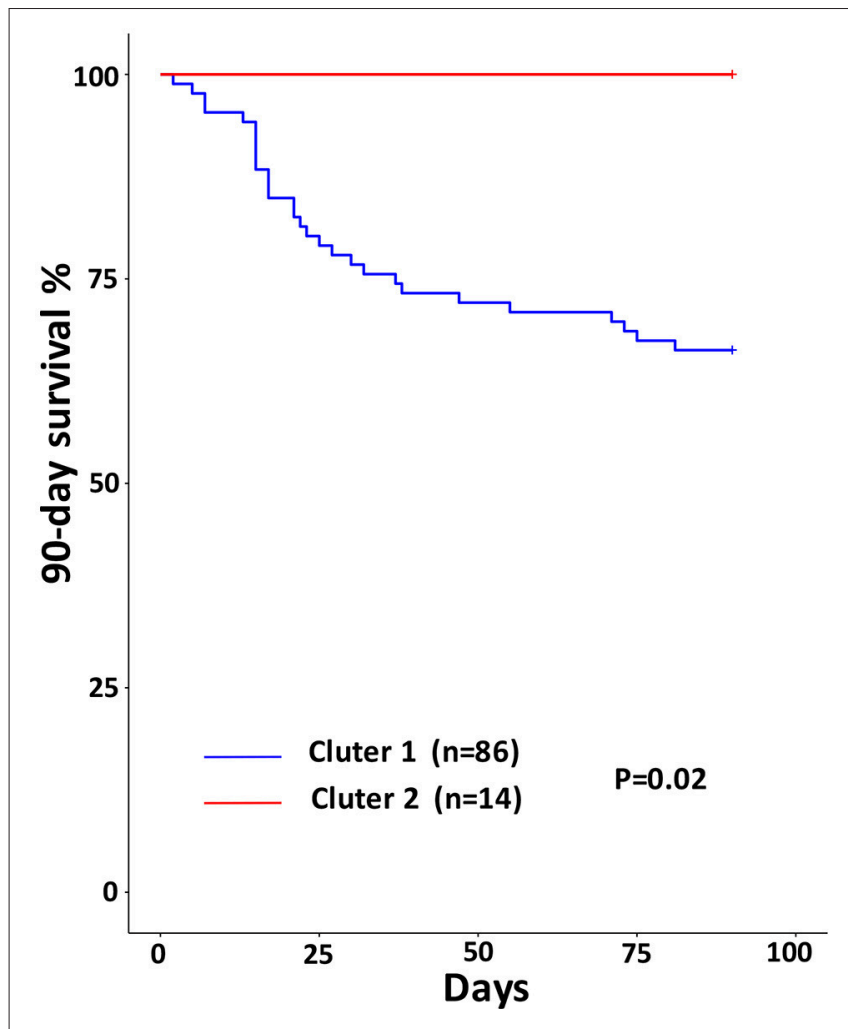

FIGURE 5 | Ninety-days Kaplan-Meier survival curves for different ascitic microbiota clusters. P-values were calculated by the Log Rank-test.

(Kim et al., 2011). Taken together, potential pathogen taxa enriched in Cluster 2 might contribute to bacterial infection in cirrhosis.

Our research found that several cytokines were upregulated in patients of Cluster 1, including IL-17A, IL-7, and PDGFBB. IL-17A is a pro-inflammatory cytokine, mainly produced by Th17 cells (Schulz et al., 2008). IL-17A plays dual roles including protection host from bacterial and fungi infections, and participating in the autoimmunity diseases (Arababadi et al., 2014). Gut microbiota is supposed to induce Th17 differentiation and thus regulate IL-17A production and functions (Douzandeh-Mobarrez and Kariminik, 2017). Decreased Firmicutes/Bacteroidetes ratio, has been reported in systemic lupus erythematosus, that is associated with increased Th17 activation and differentiation (López et al., 2016). In consistent with the previous study, the AF microbiota of Cluster 1 showed decreased Firmicutes/Bacteroidetes ratio with increased plasma IL-17A level. The results confirmed associations between gut microbiota and Th17 differentiation. IL-17A plays important roles in fighting against pathogenic microbes using several mechanisms including induction of antimicrobial peptides, such as $\alpha$-defensins, $\beta$-defensins, and lysozyme (Dixon et al., 2016; Kumar et al., 2016; Malaise et al., 2018). This might explain the relative lower prevalence of SBP in patients of Cluster 1 . However, Cluster 1 patients had significantly higher short-term mortality. IL-17A is able to up-regulate inducible nitric oxide synthase (iNOS), an enzyme that participates in the production of nitric oxide (Su et al., 2016). The importance of iNOSderived nitric oxide production in promoting BT has been evidenced experimentally in several studies. The mechanisms included that induces gastric mucosal damage, decreases the viability of rat colonic epithelial cells, directly dilates TJs in intestinal epithelial monolayers, inhibits ATP-formation and hence, increases intestinal permeability (Wiest et al., 2014).

Whether IL-17A has pathogenic and/or protective roles in the intestinal permeability is controversial. Several studies indicated that IL-17 promotes the expression of proteins, such as cellular tight-junction protein, ZO-1, in mucosal tissues that are involved with barrier function (Kinugasa et al., 2000). However, chronic and long-term production of IL-17 mediates inflammatory effects and neutrophil recruitment into the gut, could lead to long-term increases in gut permeability (Lee et al., 2015). Recently, the associations between gut permeability and IL-17 in chronic liver disease have been researched in several studies. Vega-Magana et al. analyzed the dynamic between TH1/TH2/TH17 cytokines and the integrity of the colonic mucosa in a rat model using bile duct ligation (Vega-Magaña et al., 2018). According to their results, bacterial overgrowth associated with BT is linked to the over-expression of IFN- $\gamma$, IL-4, IL-17, and mucin-2. They proposed that these molecules might facilitate the intestinal permeability through exacerbating the inflammatory process and disturbing tight junctions, leading to poor outcomes. In another study by Tedesco et al. hepatic activation of $\gamma \delta$ TCR + cells and production of IL17 by exposure to gut-derived Lactobacillus gasseri were found to participate in the process of liver disease (Tedesco et al., 2018). Increased serum levels of IL17 and intestinal permeability detected by oral administration of FITCdextran assay were confirmed in both human and animal studies. Although, the intestines permeability was not characterized here, higher level of IL-17 and 90-day mortality observed in patients with AF Cluster 1 in our research might also indicated increased intestinal permeability.

We demonstrated close correlations between S24-7 family and plasma cytokines. Bacteroidales S24-7 family is one of the substantial component of gut microbiota, which has not been successfully cultured. Multiple studies have since reported the altered abundance of $S 24-7$ family members in association with different physical conditions. S24-7 is more abundant in diabetessensitive mice fed a high-fat diet, in particular when chow is supplemented with gluco-oligosaccharides (Serino et al., 2012). In mouse model of colitis, enrichment of S24-7 was observed following treatment-induced remission of colitis in mice (Rooks et al., 2014). Some members of the $\$ 24-7$ family are targeted by innate immune system by inducing high-affinity antigen-specific IgA responses and become highly coated with IgA (Palm et al., 2014). It was proposed that bacterial species highly coated with IgA could stimulate intestinal immunity and drive intestinal disease.

Using comparative genomics analysis, a subset of $S 24-7$ family was found to have urease encoding genes (Ormerod et al., 2016). In the intestine, bacterial urease converts host-derived urea to ammonia and carbon dioxide, contributing to hyperammonemia associated neurotoxicity and encephalopathy in 
TABLE 2 | Cox regression analysis for variables and 90-day mortality.

\begin{tabular}{|c|c|c|c|c|c|c|}
\hline \multirow[t]{2}{*}{ Variable } & \multicolumn{3}{|c|}{ Univariate analysis } & \multicolumn{3}{|c|}{ Multivariate analysis } \\
\hline & HR & $95 \% \mathrm{Cl}$ & $P$-value & HR & $95 \% \mathrm{Cl}$ & $P$-value \\
\hline Ascitic microbial Cluster 1 & 0.038 & & 0.132 & & & \\
\hline MELD score & 1.07 & $1.018-1.124$ & 0.008 & 1.075 & $1.021-1.132$ & 0.006 \\
\hline TNF- $\alpha$ & 1.001 & $1.000-1.001$ & 0.014 & 1.001 & $1.000-1.001$ & 0.008 \\
\hline IL-12P70 & 1.002 & $1.000-1.003$ & 0.034 & & & \\
\hline IL-15 & 1.008 & $1.001-1.014$ & 0.015 & & & \\
\hline IL-2 & 1.004 & $1.000-1.008$ & 0.048 & & & \\
\hline IL-1 $\beta$ & 1.002 & $1.002-1.005$ & 0.04 & & & \\
\hline
\end{tabular}

HR, hazard ratio; 95\% Cl, 95\% confidence interval of hazard ratio. Calculated by univariate and multivariate Cox regression analysis, P-values considered significant (<0.05) was bold.

patients with liver disease (Shen et al., 2015). Urease is also a recognized virulence factor in both bacterial and fungal infection (Mora and Arioli, 2014). The enrichment of urease-positive species in Cluster 1, such as S 24-7 family and Lactobacillus, might suggest a possible mechanism of bacterial produced ammonia in progression of end-stage liver disease.

As indicated by Cox regression analysis, MELD score and plasma TNF- $\alpha$ level are independent predictors of 90-day mortality. Levels of TNF- $\alpha$ were found significantly higher in acute on chronic liver failure patients, especially in non-survivors (Berry et al., 2010; Mao et al., 2011). The Kaplan-Meier analysis showed significantly higher 90-day mortality in patients with AF Cluster 1 than Cluster 2. It is interesting that the MELD score and plasma TNF- $\alpha$ level were comparable between Cluster 1 and Cluster 2. We proposed that ascitic microbial cluster might be a potential parameter for mortality prediction with different mechanisms. However, Cox regression did not indicate AF Cluster 1 as a valid predictor of 90-day mortality. Further studies with larger sample size are in need.

\section{CONCLUSIONS}

Our study demonstrated that the microbial composition of AF in cirrhotic patients have correlations with short-term clinical outcomes. The possible mechanisms include intestinal permeability-dependent BT and immune responses triggered by different translocated microbes. While the present study strengthens the association between AF microbiota and clinical outcomes, it has limitations. The intestinal microbiota and intestinal permeability of the participants was not analyzed simultaneously. We were unable to identify a direct pathway

\section{REFERENCES}

Arababadi, M. K., Bidaki, M. Z., and Kennedy, D. (2014). IL-17A in hepatitis B infection: friend or foe? Arch. Virol. 159, 1883-1888. doi: $10.1007 / \mathrm{s} 00705-014-2002-\mathrm{x}$

Arumugam, M., Raes, J., Pelletier, E., Le Paslier, D., Yamada, T., Mende, D. R., et al. (2011). Enterotypes of the human gut microbiome. Nature 473, 174-180. doi: 10.1038/nature09944 among gut dysbiosis, intestinal permeability, and immune response in end-stage liver disease. Future in vivo and in vitro studies are in need to investigate the direct correlations among intestinal permeability, translocated bacteria, and immune responses.

\section{ETHICS STATEMENT}

The ethics committee of the First Affiliated Hospital, College of Medicine, Zhejiang University approved all the work on February 27th 2014, IRB ID\#2014073. All patients gave written informed consent for inclusion in the study. The data were analyzed without personal identifiers.

\section{AUTHOR CONTRIBUTIONS}

YC and JG participated in the design of the study, collected biopsy samples, performed the statistical analysis, and wrote the paper. DS and DF carried out the DNA extraction and performed 16S rRNA gene PCR amplification. CC carried out the cytokine profile analysis. LL conceived the study and participated in its design and coordination and helped to draft the manuscript. All authors read and approved the final manuscript.

\section{FUNDING}

This work was supported by the National Key Research and Development Program of China (81790631), the National Natural Science Foundation of China (81400633), Natural Science Foundation of Zhejiang Province (LY15H030012).

Bellot, P., Francés, R., and Such, J. (2013). Pathological bacterial translocation in cirrhosis: pathophysiology, diagnosis and clinical implications. Liver. Int. 33, 31-39. doi: 10.1111/liv.12021

Berry, P. A., Antoniades, C. G., Hussain, M. J., Mcphail, M. J., Bernal, W., Vergani, D., et al. (2010). Admission levels and early changes in serum interleukin-10 are predictive of poor outcome in acute liver failure and decompensated cirrhosis. Liver Int. 30, 733-740. doi: 10.1111/j.1478-3231.2010. 02219.x 
Bossola, M., Sanguinetti, M., Scribano, D., Zuppi, C., Giungi, S., Luciani, G., et al. (2009). Circulating bacterial-derived DNA fragments and markers of inflammation in chronic hemodialysis patients. Clin. J. Am. Soc. Nephrol. 4, 379-385. doi: 10.2215/CJN.03490708

Breslow, J. M., Meissler, J. J. Jr., Hartzell, R. R., Spence, P. B., Truant, A., Gaughan, J., et al. (2011). Innate immune responses to systemic Acinetobacter baumannii infection in mice: neutrophils, but not interleukin-17, mediate host resistance. Infect. Immun. 79, 3317-3327. doi: 10.1128/IAI.00069-11

Caro, E., Francés, R., Zapater, P., Pascual, S., Bellot, P., and Such, J. (2016). Grade of soluble inflammatory response is mainly affected by circulating bacterial DNA concentrations in cirrhosis. Liver Int. 36, 1473-1480. doi: 10.1111/liv.13118

De Smet, A. M., Kluytmans, J. A., Cooper, B. S., Mascini, E. M., Benus, R. F., Van Der Werf, T. S., et al. (2009). Decontamination of the digestive tract and oropharynx in ICU patients. N. Engl. J. Med. 360, 20-31. doi: 10.1056/NEJMoa0800394

Dixon, B. R., Radin, J. N., Piazuelo, M. B., Contreras, D. C., and Algood, H. M. (2016). IL-17a and IL-22 induce expression of antimicrobials in gastrointestinal epithelial cells and may contribute to epithelial cell defense against Helicobacter pylori. PLoS One 11:e0148514. doi: 10.1371/journal.pone.0148514

Douzandeh-Mobarrez, B., and Kariminik, A. (2017). Gut microbiota and IL17A: physiological and pathological responses. Probiotics Antimicrob. Proteins. doi: 10.1007/s12602-017-9329-z

Edgar, R. C., Haas, B. J., Clemente, J. C., Quince, C., and Knight, R. (2011). UCHIME improves sensitivity and speed of chimera detection. Bioinformatics 27, 2194-2200. doi: 10.1093/bioinformatics/btr381

Fagan, K. J., Rogers, G. B., Melino, M., Arthur, D. M., Costello, M. E., Morrison, M., et al. (2015). Ascites bacterial burden and immune cell profile are associated with poor clinical outcomes in the absence of overt infection. PLOS ONE 10:e0120642. doi: 10.1371/journal.pone.0120642

Feng, Y., Chen, C. L., Chen, T. H., Liang, Y. H., Chen, H. L., Lin, C. Y., et al. (2015). Application of next-generation sequencing to study ascitic microbiome in cirrhotic patients with or without spontaneous bacterial peritonitis. $J$. Microbiol. Immunol. Infect. 48, 504-509. doi: 10.1016/j.jmii.2014.07.005

Gunjaca, I., and Francetić, I. (2010). Prevalence and clinical outcome of spontaneous bacterial peritonitis in hospitalized patients with liver cirrhosis: a prospective observational study in central part of Croatia. Acta Clin. Croat. $49,11-18$.

Gutiérrez, A., Zapater, P., Juanola, O., Sempere, L., García, M., Laveda, R., et al. (2016). Gut bacterial DNA translocation is an independent risk factor of flare at short term in patients with crohn's disease. Am. J. Gastroenterol. 111, 529-540. doi: 10.1038/ajg.2016.8

Kim, Y. J., Yoon, J. H., Kim, S. I., Hong, K. W., Kim, J. I., Choi, J. Y., et al. (2011). High mortality associated with Acinetobacter species infection in liver transplant patients. Transplant. Proc. 43, 2397-2399. doi: 10.1016/j.transproceed.2011.06.011

Kinugasa, T., Sakaguchi, T., Gu, X., and Reinecker, H. C. (2000). Claudins regulate the intestinal barrier in response to immune mediators. Gastroenterology 118, 1001-1011. doi: 10.1016/S0016-5085(00)70351-9

Kumar, P., Monin, L., Castillo, P., Elsegeiny, W., Horne, W., Eddens, T., et al. (2016). Intestinal interleukin-17 receptor signaling mediates reciprocal control of the gut microbiota and autoimmune inflammation. Immunity 44, 659-671. doi: 10.1016/j.immuni.2016.02.007

Leclercq, S., Matamoros, S., Cani, P. D., Neyrinck, A. M., Jamar, F., Stärkel, P., et al. (2014). Intestinal permeability, gut-bacterial dysbiosis, and behavioral markers of alcohol-dependence severity. Proc. Natl. Acad. Sci. U.S.A. 111, E4485-4493. doi: 10.1073/pnas.1415174111

Lee, J. S., Tato, C. M., Joyce-Shaikh, B., Gulen, M. F., Cayatte, C., Chen, Y., et al. (2015). Interleukin-23-independent IL-17 production regulates intestinal epithelial permeability. Immunity 43, 727-738. doi: 10.1016/j.immuni.2015.09.003

Llovet, J. M., Bartolí, R., Planas, R., Viñado, B., Pérez, J., Cabré, E., et al. (1996). Selective intestinal decontamination with norfloxacin reduces bacterial translocation in ascitic cirrhotic rats exposed to hemorrhagic shock. Hepatology 23, 781-787. doi: 10.1002/hep.510230419

López, P., De Paz, B., Rodríguez-Carrio, J., Hevia, A., Sánchez, B., Margolles, A., et al. (2016). Th17 responses and natural IgM antibodies are related to gut microbiota composition in systemic lupus erythematosus patients. Sci. Rep. 6:24072. doi: $10.1038 /$ srep 24072
Malaise, Y., Menard, S., Cartier, C., Lencina, C., Sommer, C., Gaultier, E., et al. (2018). Consequences of bisphenol a perinatal exposure on immune responses and gut barrier function in mice. Arch. Toxicol. 92, 347-358. doi: $10.1007 / \mathrm{s} 00204-017-2038-2$

Mao, W. L., Chen, Y., Chen, Y. M., and Li, L. J. (2011). Changes of serum cytokine levels in patients with acute on chronic liver failure treated by plasma exchange. J. Clin. Gastroenterol. 45, 551-555. doi: 10.1097/MCG.0b013e3181faefa3

McMurdie, P. J., and Holmes, S. (2013). Phyloseq: an R package for reproducible interactive analysis and graphics of microbiome census data. PLOS ONE 8:e61217. doi: 10.1371/journal.pone.0061217

Mora, D., and Arioli, S. (2014). Microbial urease in health and disease. PLoS Pathog. 10:e1004472. doi: 10.1371/journal.ppat.1004472

Ormerod, K. L., Wood, D. L., Lachner, N., Gellatly, S. L., Daly, J. N., Parsons, J. D., et al. (2016). Genomic characterization of the uncultured Bacteroidales family S24-7 inhabiting the guts of homeothermic animals. Microbiome 4:36. doi: 10.1186/s40168-016-0181-2

Palm, N. W., De Zoete, M. R., Cullen, T. W., Barry, N. A., Stefanowski, J., Hao, L., et al. (2014). Immunoglobulin A coating identifies colitogenic bacteria in inflammatory bowel disease. Cell 158, 1000-1010. doi: 10.1016/j.cell.2014.08.006

Rangel-Frausto, M. S., Pittet, D., Costigan, M., Hwang, T., Davis, C. S., and Wenzel, R. P. (1995). The natural history of the systemic inflammatory response syndrome (SIRS). A prospective study. J. Am. Med. Assoc. 273, 117-123. doi: 10.1001/jama.1995.03520260039030

Rogers, G. B., Van Der Gast, C. J., Bruce, K. D., Marsh, P., Collins, J. E., Sutton, J., et al. (2013). Ascitic microbiota composition is correlated with clinical severity in cirrhosis with portal hypertension. PLoS ONE 8:e74884. doi: 10.1371/journal.pone.0074884

Rooks, M. G., Veiga, P., Wardwell-Scott, L. H., Tickle, T., Segata, N., Michaud, M., et al. (2014). Gut microbiome composition and function in experimental colitis during active disease and treatment-induced remission. ISME J. 8, 1403-1417. doi: 10.1038 /ismej.2014.3

Salcher, M. M., Pernthaler, J., and Posch, T. (2011). Seasonal bloom dynamics and ecophysiology of the freshwater sister clade of SAR11 bacteria 'that rule the waves' (LD12). ISME J. 5, 1242-1252. doi: 10.1038/ismej.2011.8

Schulz, S. M., Köhler, G., Holscher, C., Iwakura, Y., and Alber, G. (2008). IL$17 \mathrm{~A}$ is produced by Th17, gammadelta $\mathrm{T}$ cells and other CD4-lymphocytes during infection with Salmonella enterica serovar Enteritidis and has a mild effect in bacterial clearance. Int. Immunol. 20, 1129-1138. doi: 10.1093/intimm/ dxn069

Segata, N., Izard, J., Waldron, L., Gevers, D., Miropolsky, L., Garrett, W. S., et al. (2011). Metagenomic biomarker discovery and explanation. Genome Biol. 12:R60. doi: 10.1186/gb-2011-12-6-r60

Serino, M., Luche, E., Gres, S., Baylac, A., Berge, M., Cenac, C., et al. (2012). Metabolic adaptation to a high-fat diet is associated with a change in the gut microbiota. Gut 61, 543-553. doi: 10.1136/gutjnl-2011-301012

Shen, T. C., Albenberg, L., Bittinger, K., Chehoud, C., Chen, Y. Y., Judge, C. A., et al. (2015). Engineering the gut microbiota to treat hyperammonemia. J. Clin. Invest. 125, 2841-2850. doi: 10.1172/JCI79214

Su, S. A., Yang, D., Zhu, W., Cai, Z., Zhang, N., Zhao, L., et al. (2016). Interleukin17A mediates cardiomyocyte apoptosis through Stat3-iNOS pathway. Biochim. Biophys. Acta 1863, 2784-2794. doi: 10.1016/j.bbamcr.2016.08.013

Such, J., Frances, R., Munoz, C., Zapater, P., Casellas, J. A., Cifuentes, A., et al. (2002). Detection and identification of bacterial DNA in patients with cirrhosis and culture-negative, non-neutrocytic ascites. Hepatology 36, 135-141. doi: 10.1053/jhep.2002.33715

Tedesco, D., Thapa, M., Chin, C. Y., Ge, Y., Gong, M., Li, J., et al. (2018). Alterations in intestinal microbiota lead to production of interleukin 17 by intrahepatic gammadelta T-cell receptor-positive cells and pathogenesis of cholestatic liver disease. Gastroenterology 154, 2178-2193. doi: 10.1053/j.gastro.2018.02.019

Turnbaugh, P. J., Hamady, M., Yatsunenko, T., Cantarel, B. L., Duncan, A., Ley, R. E., et al. (2009). A core gut microbiome in obese and lean twins. Nature 457, 480-484. doi: 10.1038/nature 07540

Vega-Magaña, N., Delgado-Rizo, V., García-Benavides, L., Del Toro-Arreola, S., Segura-Ortega, J., Morales, A., et al. (2018). Bacterial translocation is linked to increased intestinal IFN-gamma, IL-4, IL-17, and mucin-2 in cholestatic rats. Ann. Hepatol. 17, 318-329. doi: 10.5604/01.3001.0010.8662 
Wiest, R., Lawson, M., and Geuking, M. (2014). Pathological bacterial translocation in liver cirrhosis. J. Hepatol. 60, 197-209. doi: 10.1016/j.jhep.2013.07.044

Wunderink, R. G. (2010). Welkommen to our world. Emergence of antibiotic resistance with selective decontamination of the digestive tract. Am. J. Respir. Crit. Care Med. 181, 426-427. doi: 10.1164/rccm.2009121821ED

Zapater, P., Frances, R., Gonzalez-Navajas, J. M., De La Hoz, M. A., Moreu, R., Pascual, S., et al. (2008). Serum and ascitic fluid bacterial DNA: a new independent prognostic factor in non-infected patients with cirrhosis. Hepatology 48, 1924-1931. doi: 10.1002/hep.22564
Conflict of Interest Statement: The authors declare that the research was conducted in the absence of any commercial or financial relationships that could be construed as a potential conflict of interest.

Copyright (c) 2018 Chen, Guo, Shi, Fang, Chen and Li. This is an open-access article distributed under the terms of the Creative Commons Attribution License (CC BY). The use, distribution or reproduction in other forums is permitted, provided the original author(s) and the copyright owner(s) are credited and that the original publication in this journal is cited, in accordance with accepted academic practice. No use, distribution or reproduction is permitted which does not comply with these terms. 